PPPL-3254

UC-427
RECEIVED

OCT 271997

\section{OSTI}

Intense Nonneutral Beam Propagation in a Periodic Solenoidal Field Using a Macroscopic Fluid Model with Zero Thermal Emittance

by

Ronald C. Davidson, Peter Stoltz, and Chiping Chen

August 1997
PPPL-3254 


\section{PPPL Reports Disclaimer}

This report was prepared as an account of work sponsored by an agency of the United States Government. Neither the United States Government nor any agency thereof, nor any of their employees, makes any warranty, express or implied, or assumes any legal liability of responsibility for the accuracy, completeness, or usefulness of any information, apparatus, product, or process disclosed, or represents that its use would not infringe privately owned rights. Reference herein to any specific commercial produce, process, or service by trade name, trademark, manufacturer, or otherwise, does not necessarily constitute or imply its endorsement, recommendation, or favoring by the United States Government or any agency thereof. The views and opinions of authors expressed herein do not necessarily state or reflect those of the United States Government or any agency thereof.

\section{Notice}

This report has been reproduced from the best available copy. Available in paper copy and microfiche.

Number of pages in this report: 25

U.S. Department of Energy and Department of Energy Contractors can obtain copies of this report from:

Office of Scientific and Technical Information

P.O. Box 62

Oak Ridge, TN 37831

(615) 576-8401

This report is publicly available from the:

National Technical Information Service

Department of Commerce

5285 Port Royal Road

Springfield, VA 22161

(703) $487-4650$ 


\title{
Intense Nonneutral Beam Propagation in a Periodic Solenoidal Field Using a Macroscopic Fluid Model with Zero Thermal Emittance
}

\author{
Ronald C. Davidson and Peter Stoltz \\ Princeton Plasma Physics Laboratory \\ Princeton University, Princeton, NJ 08543 \\ Chiping Chen \\ Plasma Science and Fusion Center \\ Massachusetts Institute of Technology, Cambridge, MA 02139
}

\begin{abstract}
A macroscopic fluid model is teveloped to describe the nonlinear dynamics and collective processes in an intense high-current beam propagating in the z-direction through a periodic focusing solenoidal field $B_{z}(z+S)=B_{z}(z)$, where $S$ is the axial periodicity length. The analysis assumes that space-charge effects dominate the effects of thermal beam emittance, $K r_{b}^{2} \gg \epsilon_{t h}^{2}$, and is based on the macroscopic moment-Maxwell equations, truncated by neglecting the pressure tensor and higher-order moments. Here, $K=2 N_{b} Z_{i}^{2} e^{2} / \hat{\gamma}_{b}^{3} m \beta_{b}^{2} c^{2}$ is the self-field perveance, $N_{b}$ is the number of particles per unit axial length, and $r_{b}$ is the characteristic beam radius. Assuming a thin beam with $r_{b}<<S$, azimuthally symmetric beam equilibria with $\partial / \partial t=0=\partial / \partial \theta$ are investigated, allowing for an axial modulation of the beam density $n_{b}(r, z)$ and macroscopic flow velocity $V_{r b}(r, z) \hat{\mathbf{e}}_{r}+V_{\theta b}(r, z) \hat{\mathbf{e}}_{\theta}+V_{z b}(r, z) \hat{\mathbf{e}}_{z}$ by the periodic focusing field. To illustrate the considerable flexibility of the macroscopic formalism, assuming (nearly) uniform axial flow velocity $V_{b}$ over the beam cross section, beam equilibrium properties are calculated for two examples: (a) uniform radial density profile over the interval $0 \leq r<r_{b}(z)$, and (b) an infinitesimally thin annular beam centered at $r=r_{b}(z)$. The analysis generally allows for the azimuthal flow velocity $V_{\theta b}(r, z)$ to differ from the Larmor frequency, and the model is used to calculate the (leading-order) correction $\delta V_{z b}(r, z)$ to the axial flow velocity for the step-function density profile in case (a) above.
\end{abstract}

PACS Numbers: $29.27 ; 41.75 ; 41.85$

ESTMEUTION OF THIS DOCUMENT IS UNLMMTEO 


\section{INTRODUCTION}

Periodic focusing accelerators ${ }^{1-4}$ have a wide range of applications varying from basic scientific research to industrial applications. There is growing interest in developing an improved theoretical understanding of the nonlinear dynamics, and stability and transport properties of nonneutral charged particle beams in advanced high-current accelerators ${ }^{5-7}$ for applications such as heavy ion fusion, tritium production, and nuclear waste treatment. Indeed, in many regimes of practical interest, the beam intensity (as measured by the charge density and current density) is sufficiently high that self-field effects dominate the thermal effects associated with the spread in momentum of the beam particles. A kinetic treatment of beam propagation based on the nonlinear Vlasov-Maxwell equations, ${ }^{1,8-14}$ although providing a complete description of collective processes, is often difficult to implement analytically. It is the purpose of this paper to develop a macroscopic cold-fiuid model ${ }^{15}$ that provides an adequate treatment of intense beam propagation through a periodic focusing solenoidal field in circumstances where space-charge effects dominate the effects of thermal beam emittance.

By way of background, kinetic models of intense beam propagation based on the VlasovMaxwell equations describe the nonlinear evolution of the distribution function $f_{b}(\mathbf{x}, \mathbf{p}, t)$ in the phase space $(\mathbf{x}, \mathbf{p})$ and the interaction of the beam particles with the average electric and magnetic fields, $\mathbf{E}(\mathbf{x}, t)$ and $\mathbf{B}(\mathbf{x}, t)$. On the other hand, a macroscopic fluid model of intense beam propagation describes the nonlinear evolution of bulk beam properties such as the beam density $n_{b}(\mathbf{x}, t)=\int d^{3} p f_{b}$ and average flow velocity $\mathbf{V}_{b}(\mathbf{x}, t)=n_{b}^{-1} \int d^{3} p \mathbf{v} f_{b}$, and also requires ancillary assumptions (such as negligibly small thermal emittance, or an assumed equation of state for the pressure tensor) in order to truncate the macroscopic moment equations. While not containing the detailed information on the distribution of particles in momentum space, a macroscopic fluid model does describe the evolution in configuration space of macroscopic quantities such as $n_{b}(\mathbf{x}, t)$ and $\mathbf{V}_{b}(\mathbf{x}, t)$. Such a macroscopic description is intrinsically simpler theoretically than a kinetic model which describes the evolution of the distribution function in the phase space $(\mathbf{x}, \mathbf{p})$. 


\section{DISCLAIMER}

Portions of this document may be illegible in electronic image products. Images are produced from the best available original document. 
A third type of theoretical model describing intense beam propagation can also be developed $^{10,16,17}$ in which rate equations are dervied for the nonlinear evolution of statisticallyaveraged quantities, such as the center-of-mass beam coordinates, $\langle x\rangle$ and $\langle y\rangle$, the mean-square beam radius $\left\langle r^{2}\right\rangle$, the unnormalized beam emittance $\epsilon$, etc. Here, statistical averages $\langle\cdots\rangle$ are over the distribution of beam particles in the accessible phase space. Such models for the evolution of statistically-averaged quantities have been developed and applied by Sacherer ${ }^{16}$ for the case of an elliptical cross-section beam propagating through a periodic quadrupole lattice, and by Lee and $\operatorname{Cooper}^{17}$ for an axisymmetric beam propagating through a soelnoidal focusing field. Typically, such models also require ancillary assumptions for closure of the rate equation, although for the case of a cylindrically symmetric beam propagating through a solenoidal focusing field, closure of the rate equations for the mean-square radius, $\left\langle r^{2}\right\rangle$, does occur for the class of so-called 'self-similar' density profiles considered by Lee and Cooper. ${ }^{17}$ As a general remark, such models for the evolution of statistically-averaged quantities do not follow the detailed evolution of the configurationspace dependence of the beam density $n_{b}(\mathbf{x}, t)$ and flow velocity $\mathbf{V}_{b}(\mathbf{x}, t)$.

As noted earlier, a principal purpose of this paper is to develop a macroscopic fluid model that describes the nonlinear evolution of an intense nonneutral beam propagating through a periodic solenoidal focusing field in the limit of negligibly small thermal emittance $\left(\epsilon_{t h} \rightarrow 0\right)$. Pedagogical aspects of the paper are presented in Secs. II and III for the case of a thin beam propagating in the $\mathrm{z}$-direction through the applied solenoidal focusing field $\mathbf{B}^{s o l}(\mathbf{x})$ described by Eq. (1). For the special case of time-stationary $(\partial / \partial t=0)$, azimuthallysymmetric $(\partial / \partial \theta=0)$ flow, the electrostatic self-field potential $\phi^{s}(r, z)$, beam density $n_{b}(r, z)$ and average flow velocity components $V_{r b}(r, z), V_{\theta b}(r, z)$, and $V_{z b}(r, z)$ evolve self-consistently according to Eqs. (12) - (16). The stability of the equilibrium profiles described by Eqs. (12) - (16) of course can also be investigated in such a cold-fluid model by linearizing Poisson's equation (2), the continuity equation (8), and the force-balance equation (8) for smallamplitude perturbations about the equilibrium profiles.

To test the robustness of the macroscopic fluid model, in Sec. IV we specialize to the 
class of so-called 'fixed-shape' or 'self-similar' density profiles in Eq. (19) first considered by Sacherer ${ }^{16}$ for an elliptical cross-section beam in a periodic quadrupole lattice, and by Lee and Cooper ${ }^{17}$ for an axisymmetric beam in a solenoidal focusing field. To lowest order, we assume $V_{z b}(r, z) \simeq V_{b}=$ const. over the radial cross section of the beam, and selfconsistent expressions are obtained for the equilibrium profiles for the electrostatic potential $\phi^{s}(r, z)$ [Eq. (22)], the radial flow velocity $V_{r b}(r, z)$ [Eq. (21)], and the azimuthal flow velocity [Eq. (24)], allowing for average beam rotation $\left(\omega_{b} \neq 0\right)$ relative to the Larmor frequency. The radial force balance equation (9) is used to derive (self-consistently) the envelope equation for $r_{b}(z)$ for two examples: (a) a density profile which is uniform radially over the interval $0 \leq r<r_{b}(z)$, and (b) an infinitesimally thin annular beam centered at $r=r_{b}(z)$. As would be expected, the envelope equation (28), derived for the case of a step-function density profile, is identical in the zero-thermal-emittance limit $\left(\epsilon_{t h}=0\right)$ to the result obtained by Chen, et. al. ${ }^{13}$ using a kinetic (Vlasov) model of a Kapchinskij-Vladimirskij-like distribution that includes average beam rotation relative to the Larmor frequency, and to the envelope equation derived by Lee and Cooper ${ }^{17}$ based on a consideration of the statistically-averaged rate equation for the rms beam radius including average beam rotation. Similarly, the envelope equation (33) for a thin annular beam agrees with the envelope equation derived in Ref. 17 when a similar form is assumed for the density profile. Also importantly, to illustrate the versatility of the macroscopic cold-fluid formalism, in Sec. IV the leading-order modification $\delta V_{z b}$ to the axial flow velocity $V_{z b}=V_{b}+\delta V_{z b}$ is calculated. Specifically, using the lowest-order expressions for $V_{r b}, V_{\theta b}$ and $B_{\theta}^{s}=-\partial A_{z}^{s} / \partial r$ obtained for a step-function radial density profile, the axial force balance equation (16) is used to calculate the profile for $\delta V_{z b}$ to leading order [Eq. (38) and (39)].

To summarize, the macroscopic cold-fluid model is found to be robust and flexible, and offers several advantages in analytical simplicity relative to a theoretical description based on the Vlasov-Maxwell equations. In the regard, it should be emphasized that the equilibrium equations (12) - (16) for axisymmetric flow can also be applied in circumstances where the density profile $n_{b}(r, z)$ has more general r-z dependence that that incorporated by the class 
of fixed-shape density profiles in Eq. (19). Moreover, the cold-fluid formalism developed in Secs. II and III can also be used to investigate detailed stability behavior for perturbations with $\partial / \partial \theta \neq 0$ and $\partial / \partial t \neq 0$.

\section{THEORETICAL MODEL AND ASSUMPTIONS}

We consider a thin, intense charged particle beam with characteristic radius $r_{b}$ and axial velocity $V_{b}$ propagating in the $\mathrm{z}$-direction through an applied solenoidal field ${ }^{1}$

$$
\mathbf{B}^{s o l}(\mathbf{x})=B_{z}(z) \hat{\mathbf{e}}_{z}-\frac{1}{2} r B_{z}^{\prime}(z) \hat{\mathbf{e}}_{r}
$$

Here, $r=\left(x^{2}+y^{2}\right)^{1 / 2}$ is the radial distance from the beam axis, $z$ is the axial coordinate, $B_{z}(z+S)=B_{z}(z)$ is the axial magnetic field with fundamental periodicity length $S$, prime denotes derivative with respect to $z$, and $r_{b}<<S$ is assumed in the thin-beam approximation. Consistent with the thin-beam approximation, the transverse kinetic energy of a beam particle is assumed to be small in comparison with its axial kinetic energy, and it is also assumed that $\nu / \hat{\gamma}_{b}=Z_{i}^{2} e^{2} N_{b} / \hat{\gamma}_{b} m c^{2}<1$, where $\nu$ is Budker's parameter. Here, $N_{b}=\int d x d y n_{b}$ is the number of charged particles per unit axial length, $\hat{\gamma}_{b} m c^{2}$ is the characteristic energy of a beam particle, $Z_{i} e$ is the particle charge, $m$ is the rest mass, and $c$ is the speed of light in vacuo. In the electrostatic approximation, the self electric field produced by the beam space charge is $\mathbf{E}^{s}=-\nabla \phi^{s}(x, y, z, t)$, where the electrostatic potential $\phi^{s}$ is determined self-consistently from Poisson's equation

$$
\nabla_{\perp}^{2} \phi^{s}=-4 \pi Z_{i} e n_{b}
$$

In Eq. (2), $n_{b}(x, y, z, t)$ is the particle density, and we have approximated $\nabla^{2} \simeq \nabla_{\perp}^{2}=$ $\partial^{2} / \partial x^{2}+\partial^{2} / \partial y^{2}$ in the thin-beam approximation. In addition, the axial beam current, $Z_{i} e n_{b} V_{z b}$, produces a transverse self-magnetic field, $\mathbf{B}^{s}=B_{x}^{s} \hat{\mathbf{e}}_{x}+B_{y}^{s} \hat{\mathbf{e}}_{y}=\nabla \times A_{z}^{s} \hat{\mathbf{e}}_{z}$, where $A_{z}^{s}(x, y, z, t)$ is determined self-consistently from

$$
\nabla_{\perp}^{2} A_{z}^{s}=-4 \pi Z_{i} e n_{b} V_{z b}
$$


Here, $V_{z b}(x, y, z, t)$ is the axial flow velocity. In circumstances where the axial flow velocity is approximately uniform over the beam cross section with $V_{z b} \simeq V_{b}=\beta_{b} c=$ const., a comparison of Eqs. (2) and (3) shows that the self-field potentials, $\phi^{s}$ and $A_{z}^{s}$, are related by the familiar expression ${ }^{1} A_{z}^{s}=\beta_{b} \phi^{s}$.

In many applications of practical interest, the beam intensity (as measured by the charge density and current density) is sufficiently intense that self-field effects dominate thermal effects associated with the spread in momentum of the beam particles. A useful dimensionless measure of the self-field intensity is the self-field perveance ${ }^{1}$ defined by $K=\left(2 / \hat{\gamma}_{b}^{3} \beta_{b}^{2}\right)\left(Z_{i}^{2} e^{2} N_{b} / m c^{2}\right)$, where $\hat{\gamma}_{b}=\left(1-\beta_{b}^{2}\right)^{-1 / 2}$ is the relativistic mass factor. Whenever the beam intensity is sufficiently large that

$$
K r_{b}^{2}>>\epsilon_{t h}^{2}
$$

the motion of the particles composing the beam can be characterized as approximately laminar, and described to good approximation by a macroscopic cold-fluid model. In Eq. (4), $r_{b}$ is the characteristic radius of the beam envelope, and $\epsilon_{t h}$ is the unnormalized transverse thermal emittance ${ }^{1,14}$ defined in terms of rms momentum spread relative to the mean. In circumstances where the inequality in Eq. (4) is satisfied, the phase advance $\sigma$ is highly depressed by self-field effects.

In the remainder of this paper, consistent with Eq. (4), we develop a macroscopic coldfluid or zero-thermal-emittance model to describe the nonlinear dynamics of the beam. ${ }^{15}$ In the six-dimensional phase space $(x, p)$, the distribution function for a cold beam can be expressed as

$$
f_{b}(\mathbf{x}, \mathbf{p}, t)=n_{b}(\mathbf{x}, t) \delta\left[\mathbf{p}-\gamma_{b}(\mathbf{x}, t) m \mathbf{V}_{b}(\mathbf{x}, t)\right]
$$

where $n_{b}(\mathbf{x}, t)$ is the density, $\mathbf{P}_{b}(\mathbf{x}, t)=\gamma_{b}(\mathbf{x}, t) m \mathbf{V}_{b}(\mathbf{x}, t)$ is the momentum of a fluid element, and

$$
\begin{aligned}
\gamma_{b}(\mathbf{x}, t) & =\left[1+\frac{\mathbf{P}_{b}^{2}(\mathbf{x}, t)}{m^{2} c^{2}}\right]^{1 / 2} \\
& =\left[1-\mathbf{V}_{b}^{2}(\mathbf{x}, t) / c^{2}\right]^{-1 / 2}
\end{aligned}
$$


is the relativistic mass factor of a fluid element. From Eq. (5), note that the pressure tensor $\tilde{\mathbf{P}}_{b}(\mathbf{x}, t)$ defined in the usual manner as the average flux relative to the mean of momentum relative to the mean is identically zero, i.e.,

$$
\tilde{\mathbf{P}}_{b}(\mathbf{x}, t)=\int d^{3} p\left(\mathbf{v}-\mathbf{V}_{b}\right)\left(\mathbf{p}-\mathbf{P}_{b}\right) f_{b}=0
$$

The distribution function $f_{b}(\mathbf{x}, \mathbf{p}, t)$ in Eq. (5) evolves according to the nonlinear Vlasov equation. ${ }^{15}$ Taking successive moments of the Vlasov equation gives for the evolution of $n_{b}=\int d^{3} p f_{b}$ and $n_{b} \gamma_{b} m \mathbf{V}_{b}=\int d^{3} p \mathrm{p} f_{b}$,

$$
\begin{gathered}
\frac{\partial n_{b}}{\partial t}+\frac{\partial}{\partial \mathbf{x}} \cdot\left(n_{b} \mathbf{V}_{b}\right)=0 \\
n_{b}\left(\frac{\partial}{\partial t}+\mathbf{V}_{b} \cdot \frac{\partial}{\partial \mathbf{x}}\right) \gamma_{b} m \mathbf{V}_{b}=n_{b} Z_{i} e\left(-\nabla \phi^{s}+\frac{1}{c} \mathbf{V}_{b} \times \mathbf{B}^{s}\right. \\
\left.+\frac{1}{c} \mathbf{V}_{b} \times\left[B_{z}(z) \hat{\mathbf{e}}_{z}-\frac{1}{2} B_{z}{ }^{\prime}(z) r \hat{\mathbf{e}}_{r}\right]\right),
\end{gathered}
$$

where $\mathbf{B}^{s}=\nabla \times A_{z}^{s} \hat{\mathbf{e}}_{z}$. By virtue of Eq. $(7), \nabla \cdot \tilde{\mathbf{P}}_{b}=0$ and the pressure gradient contribution to the force-balance equation (9) vanishes, thereby leading to a closure of the macroscopic fluid equations.

To briefly summarize, for a beam with intense self fields and negligibly small thermal emittance [Eq. (4)], Eqs. (2), (3), (8), and (9) provide a closed description of the nonlinear evolution of the self-field potentials, $\phi^{s}(\mathbf{x}, t)$ and $A^{s}(\mathbf{x}, t)$, the density $n_{b}(\mathbf{x}, t)$, and the flow velocity $\mathbf{V}_{b}(\mathbf{x}, t)$. Indeed, Eqs. (2), (3), (8), and (9) can be used to investigate the detailed dynamics of intense beam propagation through a periodic solenoidal focusing field described by Eq. (1) for a broad range of system parameters consistent with Eq. (4) and the thin-beam approximation described earlier in the paper. Making use of Eqs. (6) and (9), it is readily shown that

$$
n_{b}\left(\frac{\partial}{\partial t}+\mathbf{V}_{b} \cdot \frac{\partial}{\partial \mathbf{x}}\right)\left[\gamma_{b} m c^{2}+Z_{i} e \phi^{s}(\mathbf{x}, t)\right]=n_{b} Z_{i} e \frac{\partial \phi^{s}}{\partial t}
$$

which is a statement of energy balance in the present cold-fluid model of beam propagation. As expected, whenever $\partial \phi^{s} / \partial t=0$, total energy (kinetic plus electrostatic potential energy) 
is conserved. As noted earlier, it is assumed that the beam has large kinematic momentum directed predominantly in the axial direction. In cylindrical coordinates, we express $\mathbf{V}_{b}(\mathbf{x}, t)=V_{r b} \hat{\mathbf{e}}_{r}+V_{\theta b} \hat{\mathbf{e}}_{\theta}+\left(V_{b}+\delta V_{z b}\right) \hat{\mathbf{e}}_{z}$, where $V_{b}=$ const., and expand $\gamma_{b}(\mathbf{x}, t)$ for $V_{r b}, V_{\theta b}$ and $\delta V_{z b}$ small in comparison with $V_{b}$. Correct to quadratic order, this gives

$$
\gamma_{b}(\mathbf{x}, t) m c^{2}=\hat{\gamma}_{b} m c^{2}+\frac{1}{2} \hat{\gamma}_{b} m\left[V_{r b}^{2}+V_{\theta b}^{2}+2 V_{b}\left(\delta V_{z b}\right)+\left(\delta V_{z b}\right)^{2}\right]+\ldots
$$

where $\hat{\gamma}_{b} \equiv\left(1-V_{b}^{2} / c^{2}\right)^{-1 / 2}$.

\section{FOCUSED EQUILIBRIUM FLOW IN A PERIODIC SOLENOIDAL FIELD}

In the remainder of this article, we specialize to the case of time-stationary flow $(\partial / \partial t=$ 0 ) of azimuthally symmetric $(\partial / \partial \theta=0)$ beam equilibria, generally allowing for $r$-and- $z$ variations of the beam density $n_{b}(r, z)$, and flow velocity components $V_{r b}(r, z), V_{\theta b}(r, z)$, and $V_{z b}(r, z)=V_{b}+\delta V_{z b}(r, z)$. As noted earlier, for approximately uniform axial flow velocity over the beam cross section it follows from Eqs. (2) and (3) that $A_{z}^{s}(r, z)=\beta_{b} \phi^{s}(r, z)$, where $\beta_{b}=V_{b} / c$. In this case, the self-magnetic field is $\mathbf{B}^{s}=\nabla \times A_{z}^{s} \hat{\mathbf{e}}_{z}=B_{\theta}^{s}(r, z) \hat{\mathbf{e}}_{\theta}$, where $B_{\theta}^{s}=-\beta_{b} \partial \phi^{s} / \partial r$. For $\partial / \partial t=0$ and $\partial / \partial \theta=0$, it then follows from Eqs. (2), (8) and (9) that the self-field potential $\phi^{s}(r, z)$, beam density $n_{b}(r, z)$, and flow velocity components $V_{r b}(r, z), V_{\theta b}(r, z)$, and $V_{z b}(r, z)=V_{b}+\delta V_{z b}(r, z)$ solve

$$
\begin{gathered}
\frac{1}{r} \frac{\partial}{\partial r} r \frac{\partial \phi^{s}}{\partial r}=-4 \pi Z_{i} e n_{b}, \\
\frac{1}{r} \frac{\partial}{\partial r}\left(r n_{b} V_{r b}\right)+\frac{\partial}{\partial z}\left(n_{b} V_{z b}\right)=0
\end{gathered}
$$

and

$$
\begin{gathered}
n_{b}\left(V_{r b} \frac{\partial}{\partial r}+V_{z b} \frac{\partial}{\partial z}\right) V_{r b}-\frac{n_{b} V_{\theta b}^{2}}{r}=\frac{Z_{i} e n_{b}}{\hat{\gamma}_{b} m}\left[-\left(1-\frac{V_{z b}}{c} \beta_{b}\right) \frac{\partial \phi^{s}}{\partial r}+\frac{1}{c} V_{\theta b} B_{z}(z)\right] \\
n_{b}\left(V_{r b} \frac{\partial}{\partial r}+V_{z b} \frac{\partial}{\partial z}\right) V_{\theta b}+\frac{n_{b} V_{\theta b} V_{r b}}{r}=\frac{Z_{i} e n_{b}}{\hat{\gamma}_{b} m}\left[-\frac{1}{c} V_{r b} B_{z}(z)-\frac{1}{2 c} V_{z b} B_{z}^{\prime}(z) r\right]
\end{gathered}
$$




$$
n_{b}\left(V_{r b} \frac{\partial}{\partial r}+V_{z b} \frac{\partial}{\partial z}\right) V_{z b}=\frac{Z_{i} e n_{b}}{\hat{\gamma}_{b} m}\left[-\frac{\partial \phi^{s}}{\partial z}-\frac{V_{r b}}{c} \beta_{b} \frac{\partial \phi^{s}}{\partial r}+\frac{1}{2 c} V_{\theta b} B_{z}{ }^{\prime}(z) r\right]
$$

In Eqs. (13) - (16), the axial velocity $V_{z b}$ is typically large in magnitude in comparison with the transverse components $V_{r b}$ and $V_{\theta b}$. However, $V_{r b} \partial / \partial r$ and $V_{z b} \partial / \partial z$ are allowed to be of the same order.

In obtaining Eqs. (14) - (16) from Eq. (9), we have expressed $B_{\theta}^{s}=-\beta_{b} \partial \phi^{s} / \partial r$, and made use of Eq. (11) to approximate $\hat{\gamma}_{b}(\mathbf{x}, t) \simeq \hat{\gamma}_{b}$. Some straightforward algebra shows that Eq. (15) can be expressed in the equivalent form

$$
n_{b}\left(V_{r b} \frac{\partial}{\partial r}+V_{z b} \frac{\partial}{\partial z}\right)\left(r V_{\theta b}+\frac{1}{2} \Omega_{c b}(z) r^{2}\right)=0
$$

where $\Omega_{c b}(z)=Z_{i} e B_{z}(z) / \hat{\gamma}_{b} m c$ is the cyclotron frequency in the axial magnetic field $B_{z}(z)$. Note that Eq. (17) is simply a statement of the conservation of canonical angular momentum following the motion of a fluid element. Multiplying Eqs. (14), (15), and (16) by $V_{r b}, V_{\theta b}$, and $V_{z b}$, respectively, and adding, readily gives the energy conservation relation

$$
n_{b}\left(V_{r b} \frac{\partial}{\partial r}+V_{z b} \frac{\partial}{\partial z}\right)\left[\frac{1}{2} \hat{\gamma}_{b} m\left(V_{r b}^{2}+V_{\theta b}^{2}+V_{z b}^{2}\right)+Z_{i} e \phi^{s}\right]=0 .
$$

Comparing Eq. (18) with Eq. (10) when $\partial \phi^{s} / \partial t=0$, and making use of $V_{z b}=V_{b}+\delta V_{z b}$, it is clear that Eq. (18) is the expected result to the level of accuracy of the approximate expression for $\hat{\gamma}_{b}(\mathbf{x}, t)$ in Eq. (11).

To briefly summarize, for a thin beam with intense self fields and negligibly small thermal emittance, propagating through a periodic solenoidal focusing field, Eqs. (12) - (16) provide a closed description of the equilibrium flow for $\partial \phi^{s} / \partial t=0$ and $\partial \phi^{s} / \partial \theta=0$. For determination of $V_{\theta b}$, note that Eq. (15) can be replaced by Eq. (17). Moreover, either of Eqs. (14) or (16) can be replaced by the energy conservation relation in Eq. (18).

\section{EXAMPLES OF PERIODICALLY-FOCUSED BEAM EQUILIBRIA}

Evidently, Eqs. (12) - (16) can be used to investigate focused equilibrium flow in a periodic solenoidal field for a wide range of system parameters and classes of profiles for the 
beam density $n_{b}$ and flow velocity $\mathbf{V}_{b}$. For our purposes here, we illustrate the application of Eqs. (12) - (16) for the simple case where the lowest-order axial flow velocity is uniform with $V_{z b}(r, z)=V_{b}=$ const., and the density profile $n_{b}(r, z)$ has the fixed radial shape, which is modulated axially, described by ${ }^{16,17}$

$$
n_{b}(r, z)=\frac{N_{b}}{\pi r_{b}^{2}(z)} f\left(\frac{r}{r_{b}(z)}\right) .
$$

The class of 'fixed-shape' or 'self-similar' density profiles in Eq. (19) has previously been considered by Sacherer ${ }^{16}$, with appropriate generalization to the case of an elliptical beam and periodic quadrupole lattice, and by Lee and Cooper ${ }^{17}$ for the case of an axisymmetric beam and solenoidal focusing field considered here. Here, the functional form of $f\left(r / r_{b}\right)$ is yet unspecified, $N_{b}=2 \pi \int_{0}^{\infty} d r r n_{b}(r, z)=$ const. is the number of particles per unit axial length, and the normalization of $f\left(r / r_{b}\right)$ is chosen to be $\int_{0}^{\infty} d X X f(X)=1 / 2$. If, for example, $f\left(r / r_{b}\right)$ is the unit step function defined by $f\left(r / r_{b}\right)=1$ for $0 \leq r / r_{b}(z)<1$, and $f\left(r / r_{b}\right)=0$ for $r / r_{b}(z)>1$, then the density profile described by Eq. (19) has rectangular radial shape with $n_{b}=N_{b} / \pi r_{b}^{2}(z)$ for $0 \leq r<r_{b}(z)$. For general choice of $f\left(r / r_{b}\right)$ in Eq. (19), it is readily shown that the mean-square $\operatorname{radius}^{16,17}$ is $\left\langle r^{2}\right\rangle=N_{b}^{-1} 2 \pi \int_{0}^{\infty} d r r^{3} n_{b}(r, z)=$ $r_{b}^{2}(z)\left(2 \int_{0}^{\infty} d X X X^{2} f(X)\right)$. That is, apart from a constant multiplier, $r_{b}^{2}(z)$ is equal to the mean-square radius for general choice of $f\left(r / r_{b}\right)$.

We now proceed with an examination of Eqs. (12) - (16) for the case where $V_{z b}(r, z)=$ $V_{b}=$ const., and $n_{b}(r, z)$ has the form given in Eq. (19). First, substituting Eq. (19) and $V_{z b}(r)=V_{b}$ into the continuity equation (13) and integrating from $r=0$ to $r=r$ gives

$$
\begin{aligned}
r n_{b} V_{r b} & =-V_{b} \frac{\partial}{\partial z} \frac{N_{b}}{\pi} \int_{0}^{r / r_{b}(z)} d X X f(X) \\
& =r^{2} \frac{r_{b}^{\prime}(z)}{r_{b}(z)} n_{b}(r, z) V_{b} .
\end{aligned}
$$

Therefore, in the region where $n_{b} \neq 0$, Eq. (20) readily gives

$$
V_{r b}(r, z)=r \frac{r_{b}^{\prime}(z)}{r_{b}(z)} V_{b}
$$

for the radial flow velocity. Furthermore, substituting Eq. (19) into Poisson's equation (12) gives for the self-field potential 


$$
\phi^{s}(r, z)=-4 N_{b} Z_{i} e \int_{0}^{r / r_{b}(z)} \frac{d X}{X} \int_{0}^{X} d X^{\prime} X^{\prime} f\left(X^{\prime}\right),
$$

where $\phi^{s}=0$ at $r=0$ has been assumed. Note from Eq. (22) that $\phi^{s}=\phi^{s}\left(r / r_{b}(z)\right)$ for the class of density profiles described by Eq. (19). We now make use of Eq. (17), or equivalently Eq. (15), to determine the azimuthal flow velocity $V_{\theta b}(r, z)$. Substituting Eq. (21) and $V_{z b}=V_{b}$ into Eq. (17) gives

$$
n_{b}\left(V_{b} r \frac{r_{b}^{\prime}}{r_{b}} \frac{\partial}{\partial r}+V_{b} \frac{\partial}{\partial z}\right)\left(r V_{\theta b}+\frac{1}{2} \Omega_{c b}(z) r^{2}\right)=0,
$$

For smooth, differentiable $G\left(r / r_{b}(z)\right)$, it is readily shown that $\left[V_{b}\left(r r_{b}{ }^{\prime} / r_{b}\right) \partial / \partial r+\right.$ $\left.V_{b} \partial / \partial z\right] G\left(r / r_{b}\right)=0$. It therefore follows that the general solution to Eq. (23) can be expressed as

$$
r V_{\theta b}(r, z)=-\frac{1}{2} \Omega_{c b}(z) r^{2}+\omega_{b} r_{b 0}^{2} \frac{r^{2}}{r_{b}^{2}(z)} F\left(\frac{r}{r_{b}(z)}\right),
$$

where $\omega_{b} r_{b 0}^{2}=$ const. (independent of $r$ and $z$ ), and $F\left(r / r_{b}\right)$ is a yet unspecified function. Note that the term proportional to $F\left(r / r_{b}\right)$ in Eq. (24) allows the azimuthal beam rotation to differ from the Larmor frequency $-\Omega_{c b}(z) / 2$.

We now turn to the radial force balance equation (14). Substituting Eqs. (21), (24), and $V_{z b}=V_{b}$ into Eq. (14), some straightforward algebraic manipulation gives

$$
\begin{aligned}
n_{b}\left(V_{b} r \frac{r_{b}^{\prime}}{r_{b}} \frac{\partial}{\partial r}+V_{b} \frac{\partial}{\partial z}\right) r V_{b} \frac{r_{b}^{\prime}(z)}{r_{b}(z)}+n_{b} r & \left\{\left[\frac{\Omega_{c b}(z)}{2}\right]^{2}-\left[\omega_{b} \frac{r_{b 0}^{2}}{r_{b}^{2}(z)} F\left(\frac{r}{r_{b}(z)}\right)\right]^{2}\right\} \\
& =\frac{4 n_{b} N_{b} Z_{i}^{2} e^{2}}{\hat{\gamma}_{b}^{3} m r} \int_{0}^{r / r_{b}(z)} d X X f(X) .
\end{aligned}
$$

Here, use has been made of $1-\beta_{b}^{2}=1 / \hat{\gamma}_{b}^{2}$, and $V_{\theta b}$ has been expressed in terms of $r \Omega_{c b}(z) / 2$ and $F\left(r / r_{b}\right)$ by means of Eq. (24). Therefore, carrying out the derivative operations in Eq. (25) readily gives

$$
\begin{aligned}
n_{b} r\left\{V_{b}^{2} \frac{d^{2}}{d z^{2}} r_{b}(z)+\left(\left[\frac{\Omega_{c b}(z)}{2}\right]^{2}-\right.\right. & {\left.\left[\omega_{b} \frac{r_{b 0}^{2}}{r_{b}^{2}(z)} F\left(\frac{r}{r_{b}(z)}\right)\right]^{2}\right) r_{b}(z) } \\
& \left.-\frac{4 n_{b} N_{b} Z_{i}^{2} e^{2}}{\hat{\gamma}_{b}^{3} m} \frac{r_{b}(z)}{r^{2}} \int_{0}^{r / r_{b}(z)} d X X f(X)\right\}=0
\end{aligned}
$$


where $n_{b}=\left(N_{b} / \pi r_{b}^{2}\right) f\left(r / r_{b}\right)$ is the beam density.

The radial force balance equation (26), valid at each value of $r$ and $z$, relates selfconsistently the density shape function $f\left(r / r_{b}\right)$, the effective rms beam radius $r_{b}(z)$ [see discussion following Eq. (19)], and the (yet unspecified) function $F\left(r / r_{b}\right)$. At this writing, consistent with the assumptions that the axial velocity profile is uniform $\left(V_{z b}=V_{b}\right)$ in lowest order, and that the density profile has the fixed radial shape at each value of $z$ described by Eq. (19), only two solutions for $f\left(r / r_{b}\right)$ have been determined. One corresponds to a step function density profile extending from $r=0$ to $r=r_{b}(z)$. The other corresponds to a thin annular layer centered at $r=r_{b}(z)$. We discuss these two cases separately.

Step-Function Density Profile: As a first example, we consider the case where

$$
f\left(\frac{r}{r_{b}(z)}\right)=\left\{\begin{array}{l}
1,0 \leq r<r_{b}(z), \\
0, r>r_{b}(z) .
\end{array}\right.
$$

In this case, it follows from Eq. (19) that $n_{b}(r, z)=N_{b} / \pi r_{b}^{2}(z)$ in the beam interior, and that $\int_{0}^{r / r_{b}(z)} d X X f(X)=r^{2} / 2 r_{b}^{2}(z)$ for $0 \leq r<r_{b}(z)$. Substituting Eq. (27) into Eq. (26), we find that Eq. (26) is satisfied for all $r$ and $z$ provided $F\left(r / r_{b}\right)=$ const. (which we take to be unity, without loss of generality), and the outer beam radius satisfies the envelope equation

$$
V_{b}^{2} \frac{d^{2}}{d z^{2}} r_{b}(z)+\left(\frac{\Omega_{c b}^{2}(z)}{4}-\frac{\omega_{b}^{2} r_{b 0}^{4}}{r_{b}^{4}(z)}\right) r_{b}(z)-\frac{2 N_{b} Z_{i}^{2} e^{2}}{\hat{\gamma}_{b}^{3} m r_{b}(z)}=0
$$

Here, $2 N_{b} Z_{i}^{2} e^{2} / \hat{\gamma}_{b}^{3} m=V_{b}^{2} K$, where $K$ is the self-field perveance defined in the usual manner. Equation (28) is similar in form to the familiar envelope equation ${ }^{11,17}$ for a KapchinskijVladimirskij beam distribution ${ }^{8}$ in a periodic solenoidal field, assuming zero transverse thermal emittance $\left(\epsilon_{t h}=0\right)$, and including the effects of an average azimuthal beam rotation ${ }^{13,17}$ of $\omega_{b} r_{b 0}^{2} / r_{b}^{2}$ relative to the Larmor frequency $-\Omega_{c b}(z) / 2$. Not surprisingly, the envelope equation (28) is identical to the $\epsilon_{t h}=0$ limit of the envelope equation derived using a kinetic (Vlasov) model ${ }^{13}$ of a KV-like distribution that includes beam rotation $\left(\omega_{b} \neq 0\right)$ in the Larmor frame, as well as the corresponding limit (for a step-function density profile) of the envelope equation derived by Lee and Cooper ${ }^{17}$ by examination of the rate equation for the 
(statistically-averaged) $\mathrm{rms}$ beam radius $\left\langle r^{2}\right\rangle^{1 / 2}$. From Eq. (24), for $F\left(r / r_{b}\right)=1$ we find that the angular rotation velocity of the beam can be expressed for $0 \leq r<r_{b}(z)$ as

$$
\Omega_{b}(r, z) \equiv \frac{V_{\theta b}(r, z)}{r}=-\frac{1}{2} \Omega_{c b}(z)+\omega_{b} \frac{r_{b 0}^{2}}{r_{b}^{2}(z)}
$$

Note that the term proportional to $\omega_{b}^{2} r_{b 0}^{4} / r_{b}^{3}(z)$ in Eq. (28) plays the role of an effective emittance $^{1}$ contribution to the envelope equation associated with the directed azimuthal motion relative to the Larmor frequency $-\Omega_{c b}(z) / 2$. Moreover, the canonical angular momentum of a fluid element is given in terms of the parameter $\omega_{b} r_{b 0}^{2}$ by $P_{\theta b}(r, z)=\hat{\gamma}_{b} m \omega_{b} r_{b 0}^{2} r^{2} / r_{b}^{2}(z)$. In particular, note that $P_{\theta b}\left(r_{b}(z), z\right)=\hat{\gamma}_{b} m \omega_{b} r_{b 0}^{2}$ at $r=r_{b}(z)$.

Thin Annular Beam: As a second example, we consider the case where the density profile corresponds to an infinitesimally thin annulus centered at $r=r_{b}(z)$ with

$$
f\left(r / r_{b}\right)=\frac{1}{2} \delta\left(r / r_{b}-1\right)
$$

and

$$
n_{b}(r, z)=\frac{N_{b}}{2 \pi r_{b}(z)} \delta\left[r-r_{b}(z)\right]
$$

We substitute Eqs. (30) and (31) into Eq. (26) and operate on Eq. (26) with $2 \pi \int_{0}^{\infty} d r \ldots \ldots$ Taking $F(1)=1$ without loss of generality, and making use of

$$
\frac{1}{2} \int_{0}^{\infty} \frac{d r}{r^{2}} r \delta\left(r-r_{b}\right) \int_{0}^{r} d r r \delta\left(r-r_{b}\right)=\frac{1}{4} \int_{0}^{\infty} \frac{d r}{r^{2}} \frac{\partial}{\partial r}\left(\int_{0}^{r} d r r \delta\left(r-r_{b}\right)\right)^{2}=\frac{1}{4},
$$

this operation on Eq. (26) readily gives

$$
V_{b}^{2} \frac{d^{2}}{d z^{2}} r_{b}(z)+\left(\frac{\Omega_{c b}^{2}(z)}{4}-\frac{\omega_{b}^{2} r_{b 0}^{4}}{r_{b}^{4}(z)}\right) r_{b}(z)-\frac{N_{b} Z_{i}^{2} e^{2}}{\hat{\gamma}_{b}^{3} m r_{b}(z)}=0 .
$$

Equation (33) describes the axial modulation of the radius $r_{b}(z)$ of the annulus by the periodic solenoidal field $\Omega_{c b}(z)$. While similar in overall form to the envelope equation (28) for a beam with step-function density profile extending from $r=0$ to $r=r_{b}(z)$, note that the final term in Eq. (33) (associated with self-field effects) differs by a factor of two from the final term in Eq. (28). This is associated with the fact that the radial self-electric field 
is $E_{r}^{s}=-\partial \phi^{s} / \partial r=0$ for $r<r_{b}(z)$ for the annular beam described by Eq. (30), whereas $E_{r}^{s}=-\partial \phi^{s} / \partial r=-2 N_{b} Z_{i} e r / r_{b}^{2}(z)$ is non-zero for $r<r_{b}(z)$ for the step-function density profile in Eq. (27). The discontinuity in the radial self-field force when Eq. (26) is integrated across the annulus at $r=r_{b}(z)$ then accounts for the difference by a factor of two in the self-field terms in Eqs. (28) and (33). While the example of a thin annular beam is not of particular interest for the advanced high-current accelerators envisioned for heavy ion fusion and tritium production, it does serve to illustrate the power of the macroscopic formalism for intense beam propagation developed in this paper.

Determination of $\delta V_{z b}$ : We now return to the example of the step-function density profile considered in Eq. (27), and the corresponding equation for the beam envelope $r_{b}(z)$ in Eq. (28), and the expression for the angular velocity in Eq. (29). In deriving these results we have assumed that the axial flow velocity is uniform over the beam cross section with $V_{z b}=V_{b}=$ const. We now make use of the axial force balance equation (16) to calculate the leading-order correction to the axial flow velocity. [The energy balance equation (18) could also be used for this purpose.] We set $V_{z b}=V_{b}+\delta V_{z b}$ on the left-hand side of Eq. (16), and make use of Eqs. (21) and (29) to express $V_{r b}=V_{b} r r_{b}^{\prime}(z) / r_{b}(z)$ and $V_{\theta b}=$ $-\Omega_{c b}(z) r / 2+\omega_{b} r_{b 0}{ }^{2} r / r_{b}{ }^{2}(z)$ correct to leading order. Furthermore, substituting Eq. (27) into Eq. (22) gives $\phi^{s}=-N_{b} Z_{i} e r^{2} / r_{b}^{2}(z)$ for $0 \leq r<r_{b}(z)$. After some algebraic manipulation and rearrangement of terms, Eq. (16) then becomes to lowest order

$$
\begin{aligned}
n_{b}\left(V_{b} r \frac{r_{b}{ }^{\prime}}{r_{b}} \frac{\partial}{\partial r}+V_{b} \frac{\partial}{\partial z}\right) \delta V_{z b}= & n_{b}\left\{-\frac{2 N_{b} Z_{i}^{2} e^{2}}{\hat{\gamma}_{b}^{3} m} \frac{r_{b}^{\prime}(z)}{r_{b}(z)}\right. \\
& \left.+\frac{1}{2} \Omega_{c b}{ }^{\prime}(z)\left[\omega_{b}^{2} r_{b 0}^{2}-\frac{1}{2} \Omega_{c b}(z) r_{b}^{2}(z)\right]\right\} \frac{r^{2}}{r_{b}^{2}(z)} .
\end{aligned}
$$

Here, use has been made of $\hat{\gamma}_{b}{ }^{-2}=1-\beta_{b}{ }^{2}$, and $r_{b}(z)$ solves the nonlinear envelope equation (28). By inspection, the particular solution to Eq. (34) is of the form

$$
\delta V_{z b}=V_{b} f(z) \frac{r^{2}}{r_{b}^{2}(z)},
$$

where 


$$
V_{b}^{2} \frac{d}{d z} f(z)=-\frac{2 N_{b} Z_{i}^{2} e^{2}}{\hat{\gamma}_{b}^{3} m} \frac{r_{b}^{\prime}(z)}{r_{b}(z)}+\frac{1}{2} \Omega_{c b}^{\prime}(z)\left[\omega_{b}^{2} r_{b 0}^{2}-\frac{1}{2} \Omega_{c b}(z) r_{b}^{2}(z)\right]
$$

In obtaining Eqs. (35) and (36), use has been made of $\left[V_{b}\left(r r_{b}^{\prime} / r_{b}\right) \partial / \partial r+V_{b} \partial / \partial z\right] r^{2} / r_{b}^{2}(z)=0$. Multiplying the envelope equation (28) by $d r_{b} / d z$, it is readily shown that

$$
\frac{d}{d z}\left\{\frac{1}{2} V_{b}^{2}{r_{b}}^{2}+\frac{1}{2} r_{b}^{2}\left(\frac{\Omega_{c b}}{2}\right)^{2}-\frac{1}{2} \frac{\omega_{b}^{2} r_{b 0}^{4}}{r_{b}^{2}}\right\}=\frac{1}{2} r_{b}^{2}\left(\frac{\Omega_{c b}^{2}}{4}\right)^{\prime}+\frac{2 N_{b} Z_{i}^{2} e^{2}}{\hat{\gamma}_{b}^{3} m} \frac{r_{b}^{\prime}}{r_{b}} .
$$

Substituting Eq. (37) into Eq. (36) and integrating with respect to $z$, we obtain the closed expression

$$
V_{b}^{2} f(z)=\text { const. }+\frac{1}{2}\left\{\omega_{b} r_{b 0}^{2} \Omega_{c b}(z)-V_{b}^{2}{r_{b}^{\prime}}^{2}(z)-r_{b}^{2}(z)\left[\frac{\Omega_{c b}(z)}{2}\right]^{2}+\frac{\omega_{b}^{2} r_{b 0}^{4}}{r_{b}^{2}(z)}\right\}
$$

Here, $r_{b}(z)$ is determined by integrating Eq. (28) numerically, and the constant in Eq. (38) can be evaluated by setting $f(z=0)=0$ (say). Also keep in mind that Eqs. (35) and (38) have been derived for the case where the density profile corresponds to the step-function in Eq. (27).

To summarize, correct to first order, the solution for the axial flow velocity for $0 \leq r<$ $r_{b}(z)$ is

$$
V_{z b}=V_{b}\left[1+f(z) \frac{r^{2}}{r_{b}^{2}(z)}\right]
$$

where $f(z)$ is defined in Eq. (38). Note from Eq. (39) that $\delta V_{z b}=0$ at $r=0$, whereas $\delta V_{z b}$ is largest near the outer edge of the beam. From Eq. (38), the characteristic maximum value of $\delta V_{z b}$ is

$$
\frac{\left|\delta V_{z b}\right|_{\max }}{V_{b}} \sim \frac{\Omega_{c b}^{2} r_{b}^{2}}{8 V_{b}^{2}}, \frac{r_{b}^{2}}{2 S^{2}}
$$

For example, if $r_{b} / S \sim 10^{-2}$, then Eq. (40) gives $\left|\delta V_{z b}\right|_{\max } \sim 0.5 \times 10^{-4} V_{b}$, corresponding to a very small deviation in the axial flow velocity from the constant value $V_{b}$. For a periodic field with $\Omega_{c b}(z+S)=\Omega_{c b}(z)$, and a matched beam with $r_{b}(z+S)=r_{b}(z)$, we note from Eqs. (38) and (39) that the modulation of the axial flow is also periodic with $\delta V_{z b}(r, z+S)=\delta V_{z b}(r, z)$ at each radial location $r$ over the beam cross section. As a final point regarding Eqs. (38) 
and (39), it is important to note that the expressions for $V_{r b}, V_{\theta b}$ and $B_{\theta}^{s}=-\partial A_{z}^{s} / \partial r$ are only required to lowest order in order to determine the leading-order $\delta V_{z b}$ from Eq. (16). To calculate the next higher-order corrections to $V_{r b}, V_{\theta b}$ and $\delta V_{z b}$, however, it is necessary to revisit the Maxwell equation (3) and calculate $A_{z}^{s}$ to higher accuracy than $A_{z}^{s}=\beta_{b} \phi^{s}$, which assumes $V_{z b}=V_{b}=$ const. This is accomplished (in an iterative sense) by substituting $V_{z b}=V_{b}+\delta V_{z b}$ into Eq. (3), where $\delta V_{z b}$ is the lowest-order correction calculated in Eq. (39), thereby permitting a determination of $\delta B_{\theta}^{s}=4 \pi Z_{i} e r^{-1} \int_{0}^{r} d r r \delta V_{z b} n_{b}$.

Properties of Beam Envelope Equation: Finally, for completeness, we examine briefly properties of the beam envelope equation (28) for $r_{b}(z)$, derived for the case of the step-function density profile in Eq. (27). Scaling Eq. (28) by $V_{b}^{-2}$, the envelope equation for $r_{b}(z)$ can be expressed in the more familiar form ${ }^{1}$

$$
\frac{d^{2}}{d z^{2}} r_{b}(z)+\left(\kappa_{z}(z)-\frac{K}{r_{b}^{2}(z)}\right) r_{b}(z)=\frac{\hat{\omega}_{b}^{2}}{r_{b}^{3}(z)}
$$

Here, we have introduced the solenoidal focusing coefficient $\kappa_{z}(z) \equiv\left[Z_{i} e B_{z}(z) / 2 \hat{\gamma}_{b} m c V_{b}\right]^{2}$, and the self-field perveance $K \equiv 2 N_{b} Z_{i}^{2} e^{2} / \hat{\gamma}_{b}^{3} m V_{b}^{2}$. Moreover, $\hat{\omega}_{b} \equiv \omega_{b} r_{b 0}^{2} / V_{b}$ plays the role of an unnormalized beam emittance associated with the directed azimuthal motion [the term proportional to $\omega_{b}^{2}$ in Eq. (28)] relative to the Larmor frequency $-\Omega_{c b}(z) / 2$ [see Eq. (29)]. In the special case of a uniform solenoidal field with $\kappa_{z}(z)=\bar{\kappa}_{z}=$ const. (independent of $z$ ), Eq. (41) can readily be solved for the equilibrium beam radius (denoted by $r_{b s}$ ) in the smooth-beam approximation $\left(d^{2} r_{b s} / d s^{2}=0\right)$. This gives

$$
r_{b s}^{2}=\frac{K}{2 \bar{\kappa}_{z}}+\left[\left(\frac{K}{2 \bar{\kappa}_{z}}\right)^{2}+\frac{\hat{\omega}_{b}^{2}}{\bar{\kappa}_{z}}\right]^{1 / 2} .
$$

Note that $r_{b s}$ increases as the self-field intensity increases (increasing $\mathrm{K}$ ), the beam rotation increases (increasing $\hat{\omega}_{b}$ ), or the focusing strength decreases (decreasing $\bar{\kappa}_{z}$ ). Whenever $\hat{\omega}_{b}=0$, we find from Eq. (42) that $r_{b s}^{2}=K / \bar{\kappa}_{z}$, corresponding to an exact balance of the (defocusing) self-field force and the (focusing) magnetic force on a fluid element.

The more interesting case of a periodic solenoidal focusing field is illustrated in Figs. 1 and 2 , where $\kappa_{z}(z)$ is assumed to have the form of a periodic step-function lattice defined (over one lattice period) by 


$$
\kappa_{z}(z)=\left\{\begin{array}{cl}
\kappa_{z 0}=\text { const. }, & -\eta / 2 \leq z / S<\eta / 2, \\
0, & \eta / 2 \leq z / S<1-\eta / 2,
\end{array}\right.
$$

where $\eta(<1)$ is the filling factor. Equation (41) has been solved numerically for the case of a matched beam with $r_{b}(z+S)=r_{b}(z)$. Typical numerical results are illustrated in Fig. 1 where $r_{b}(z)$ is plotted versus $z$ for the choice of system parameters $S \sqrt{\kappa_{z 0}}=2.5$, $\eta=0.5, K=0.6 \times 10^{-3}$, and several values of the rotation parameter $\hat{\omega}_{b} / S$ ranging from 0 to $5 \times 10^{-3}$. Note from Fig. 1 that the beam envelope is strongly modulated as a function of $\mathrm{z}$ by the periodic solenoidal field. In addition, as expected, the average beam radius $\bar{r}_{b}=S^{-1} \int_{s_{0}}^{s_{0}+S} d z r_{b}(z)$ increases as the rotational parameter $\hat{\omega}_{b} / S$ is increased. Figure 2 shows plots of $r_{b}(z)$ versus $z$ for the choice of system parameters $S \sqrt{\kappa_{z 0}}=2.5, \eta=0.5$, $\hat{\omega}_{b} / S=10^{-4}$, and several values of the self-field perveance $K$ ranging from $10^{-4}$ to $0.6 \times 10^{-3}$. Evidently, the average beam radius $\bar{r}_{b}$ also increases as $K$ is increased.

\section{CONCLUSIONS}

In conclusion, a macroscopic cold-fluid model has been developed to describe the properties of intense nonneutral beam propagation in a periodic focusing solenoidal field assuming

that space-charge effects dominate the effects of thermal beam emittance $\left(K r_{b}^{2}>>\epsilon_{t h}^{2}\right)$. The model is found to be robust and flexible, and offers several advantages in analytical simplicity relative to a theoretical description based on the Vlasov-Maxwell equations. In this regard, it should be emphasized that this cold-fluid formalism can also be applied to circumstances where $\partial / \partial \theta \neq 0$ and the equilibrium density profile is not restricted to have the simple form in Eq. (19).

\section{Acknowledgements}

This research was supported by the U.S. Department of Energy and in part by the Office of Naval Research. 


\section{REFERENCES}

${ }^{1}$ R. C. Davidson, Physics of Nonneutral Plasmas (Addison-Wesley Publishing Co., Reading, MA, 1990), Chapter 10, and references therein.

${ }^{2}$ D. A. Edwards and M. J. Syphers, An Introduction to the Physics of High Energy Accelerators (John Wiley \& Sons, Inc., New York, 1993).

${ }^{3}$ M. Reiser, Theory and Design of Charged Particle Beams (John Wiley \& Sons, Inc., New York, 1994).

${ }^{4}$ J. D. Lawson, The Physics of Charged-Particle Beams (Oxford Science Publications, New York, 1988), and references therein.

${ }^{5}$ R. W.Muller, in Nuclear Fusion by Inertial Confinement: A Comprehensive Treatise, edited by G. Velarde, Y. Ronen, and J. M. Martinez-Val, (Chemical Rubber Co., Boca Raton, FL, 1993).

${ }^{6}$ E. P. Lee and J. Hovingh, Fusion Technology 15, 369 (1989).

${ }^{7}$ R. A. Jameson, 1993, in Advanced Accelerator Concepts, edited by J. S. Wurtele, American Institute of Physics Conference Proceedings 279(American Institute of Physics, New York), p. 969.

${ }^{8}$ I. Kapchinskij and V. Vladimirskij, in Proceedings of the International Conference on High Energy Accelerators and Instrumentation (CERN Scientific Information Service, Geneva, 1959), p. 274.

${ }^{9}$ I. Hofmann, L. Laslett, L. Smith, and I. Haber, Particle Accelerators 13, 145 (1983).

${ }^{10} \mathrm{~J}$. Struckmeier and I. Hofmann, Particle Accelerators 39, 219 (1992).

${ }^{11}$ C. Chen and R. C. Davidson, Physical Review E 49, 5679 (1994).

${ }^{12}$ Q. Qian and R. C. Davidson, Physical Review E 53, 5349 (1996). 
${ }^{13}$ C. Chen, R. Pakter, and R. C. Davidson, Physical Review Letters 79, 225 (1997).

${ }^{14}$ R. C. Davidson and C. Chen, Vlasov Equilibrium Description of Intense Nonneutral Ion Beam Propagation through a Periodic Solenoidal Focusing Field, 1997, submitted to Physics Reports.

${ }^{15}$ See, for example, Chap. 2 of Ref. 1.

${ }^{16}$ F. J. Sacherer, IEEE Transactions on Nuclear Science NS-18, 1105 (1971).

${ }^{17}$ E. P. Lee and R. K. Cooper, Particle Accelerators 7, 83 (1976). 


\section{Figure Captions}

Fig. 1. Plot of the beam envelope $r_{b}(z)$ versus axial coordinate $z$ obtained numerically from Eq. (41) for the choice of periodic step-function lattice in Eq. (43). System parameters correspond to $S \sqrt{\kappa_{z 0}}=2.5, \eta=0.5, K=0.6 \times 10^{-3}$, and $\hat{\omega}_{b} / S=0.0$ (dashed curve), $2.5 \times 10^{-3}$ (dotted curve), and $5 \times 10^{-3}$ (solid curve). The quantities $r_{b}(z)$ and $z$ are scaled by the multiplier $S^{-1}$. The periodic step function at the bottom of the graph represents (in arbitrary units) $\kappa_{z}(z)$ in Eq. (43).

Fig. 2. Plot of the beam envelope $r_{b}(z)$ versus axial coordinate $z$ obtained numerically from Eq. (41) for the choice of periodic step-function lattice in Eq. (43). System parameters correspond to $S \sqrt{\kappa_{z 0}}=2.5, \eta=0.5, \hat{\omega}_{b} / S=1.0 \times 10^{-4}$, and $K=1.0 \times 10^{-4}$ (dashed curve), $3.0 \times 10^{-4}$ (dotted curve), and $6.0 \times 10^{-4}$ (solid curve). The quantities $r_{b}(z)$ and $z$ are scaled by the multiplier $S^{-1}$. The periodic step function at the bottom of the graph represents (in arbitrary units) $\kappa_{z}(z)$ in Eq. (43). 


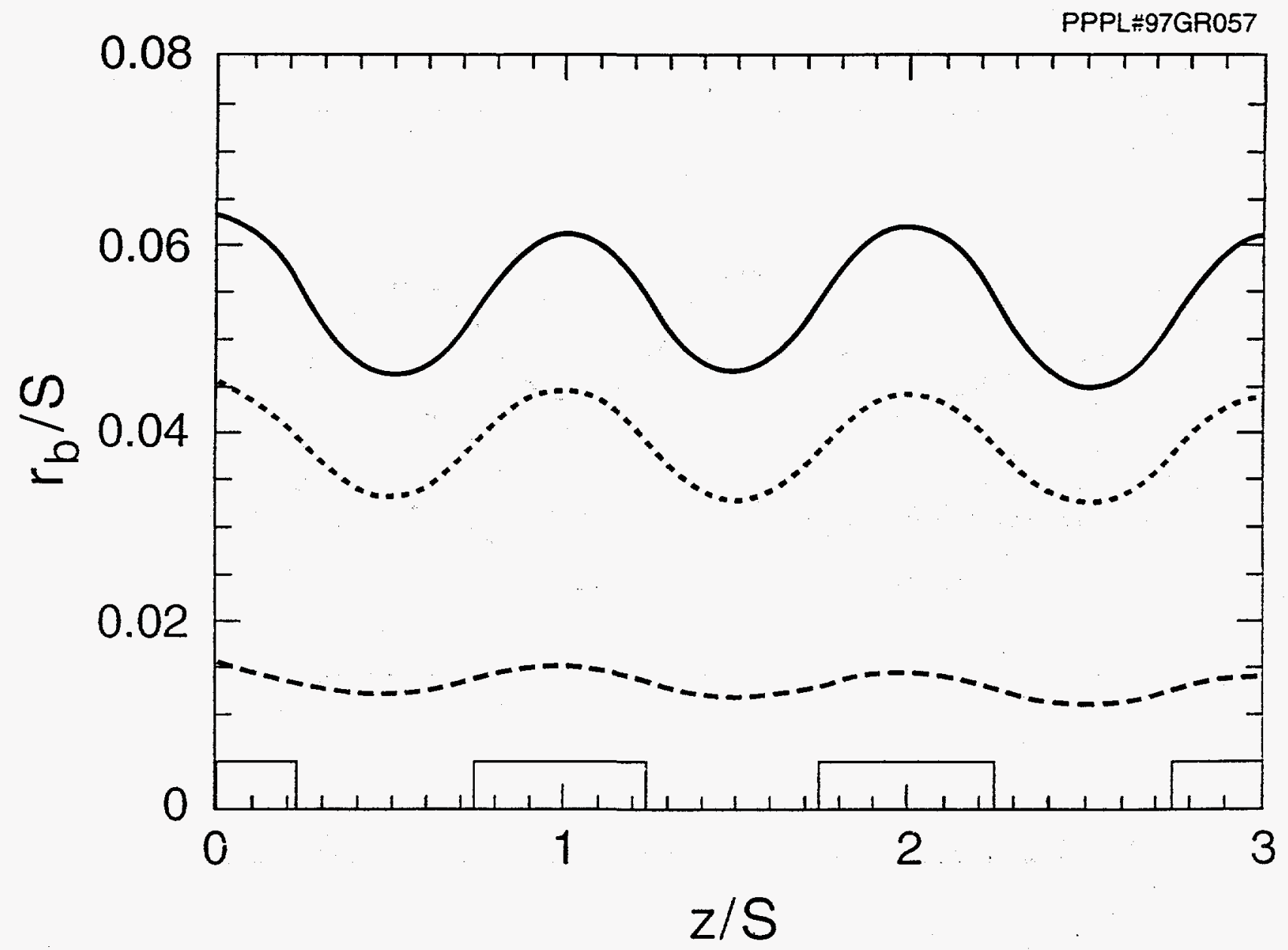

Fig. 1 


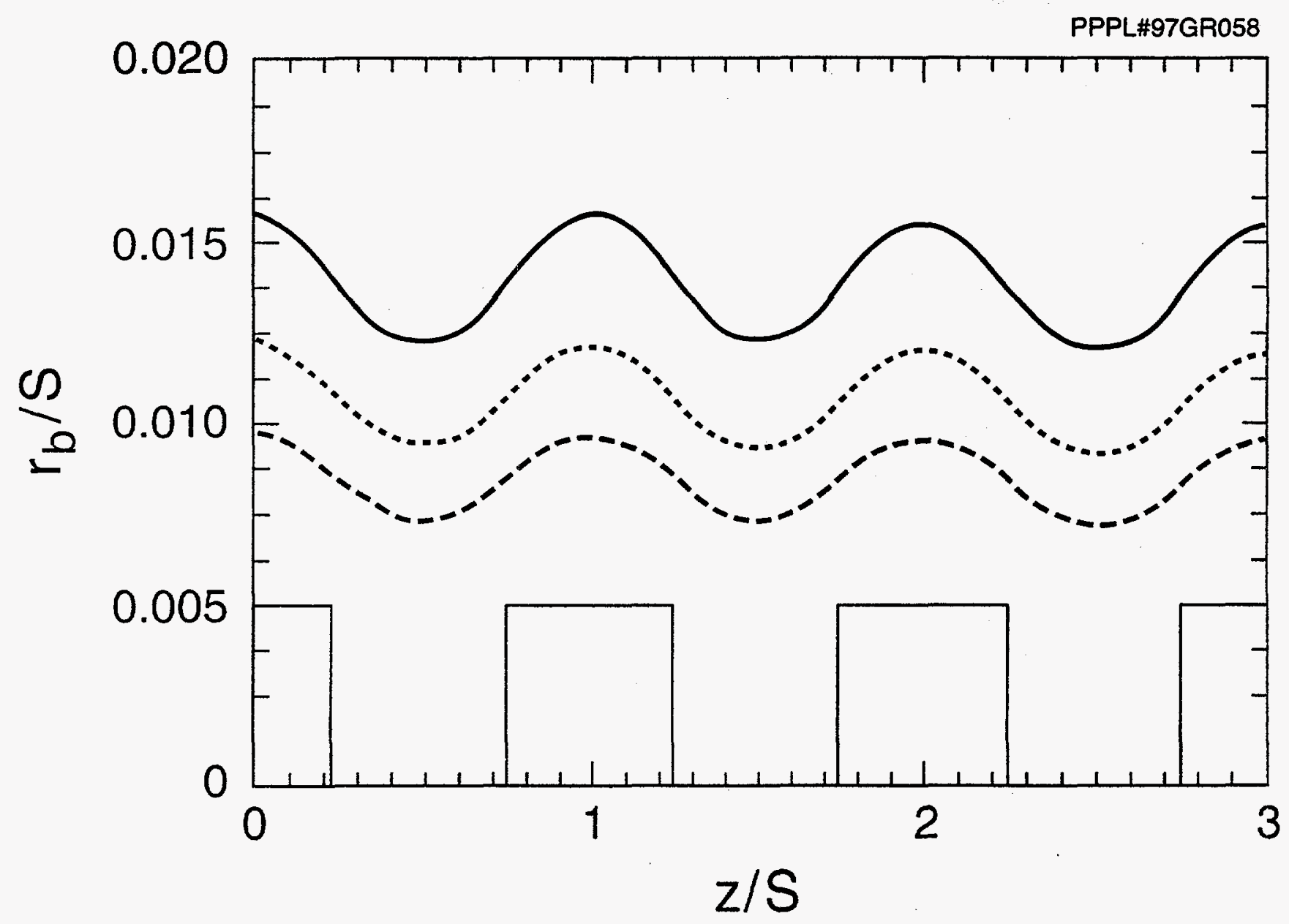

Fig. 2 


\section{External Distribution in Addition to UC-420}

Professor Joao Canalle, Instituto de Fisica DEQ/IF - UERJ, Brazil

Mr. Gerson O. Ludwig, Instituto Nacional de Pesquisas, Brazil

Dr. P.H. Sakanaka, Instituto Fisica, Brazil

Library, R61, Rutherford Appleton Laboratory, England

The Librarian, Culham Laboratory, England

Professor M.N. Bussac, Ecole Polytechnique, France

Dr. F. Moser, Bibliothek, Institute für Plasmaforschung der Universitüt Stuttgart, Germany Jolan Moldvai, Reports Library, MTA KFKI-ATKI, Hungary

Ms. Clelia de Palo, Associazione EURATOM-ENEA, Italy

Dr. G. Grosso, Instituto di Fisica del Plasma, Italy

Librarian, Naka Fusion Research Establishment, JAERI, Japan

Library, Plasma Physics Laboratory, Kyoto University, Japan

Dr. O. Mitarai, Kyushu Tokai University, Japan

Library, Academia Sinica, Insitute of Plasma Physics, People's Republic of China

Shih-Tung Tsai, Insitute of Physics, Chinese Academy of Sciences, People's Republic of China

Dr. S. Mirnov, Triniti, Troitsk, Russian Federation, Russia

Dr. V.S. Strelkov, Kurchatov Institute, Russian Federation, Russia

Mr. Dennis Bruggink, Fusion Library, University of Wisconsin, USA

Alkesh Punjabi, Center for Fusion Research and Training, Hampton University, USA

Dr. W.M. Stacey, Fusion Research Center, Georgia Institute of Technology, USA

Mr. Paul H. Wright, Indianapolis, Indiana, USA 